\title{
Dysregulation of sonic hedgehog pathway and pericytes in the brain after lentiviral infection
}

\author{
Diana G. Bohannon ${ }^{1}$, Allen Ko ${ }^{1}$, Adam R. Filipowicz' ${ }^{1}$ Marcelo J. Kuroda ${ }^{2}$ and Woong-Ki Kim
}

\begin{abstract}
Background: Impairment of the blood-brain barrier (BBB) has been associated with cognitive decline in many CNS diseases, including HIV-associated neurocognitive disorders (HAND). Recent research suggests an important role for the Sonic hedgehog (Shh) signaling pathway in the maintenance of BBB integrity under both physiological and pathological conditions.
\end{abstract}

Methods: In the present study, we sought to examine the expression of Shh and its downstream effectors in relation to brain pericytes and BBB integrity in HIV-infected humans and rhesus macaques infected with simian immunodeficiency virus (SIV), an animal model of HIV infection and CNS disease. Cortical brain tissues from uninfected $(n=4)$ and SIV-infected macaques with (SIVE, $n=6)$ or without encephalitis (SIVnoE, $n=4$ ) were examined using multi-label, semi-quantitative immunofluorescence microscopy of Shh, netrin-1, tight junction protein zona occludens 1 (ZO1), glial fibrillary acidic protein, CD163, platelet-derived growth factor receptor b (PDGFRB), glucose transporter 1, fibrinogen, and SIV Gag p28.

Results: While Shh presence in the brain persisted during HIV/SIV infection, both netrin-1 immunoreactivity and the size of PDGFRB+ pericytes, a cellular source of netrin-1, were increased around non-lesion-associated vessels in encephalitis compared to uninfected brain or brain without encephalitis, but were completely absent in encephalitic lesions. Hypertrophied pericytes were strongly localized in areas of fibrinogen extravasation and showed the presence of intracellular SIVp28 and HIVp24 by immunofluorescence in all SIV and HIV encephalitis cases examined, respectively.

Conclusions: The lack of pericytes and netrin-1 in encephalitic lesions, in line with downregulation of ZO1 on the fenestrated endothelium, suggests that pericyte loss, despite the strong presence of Shh, contributes to HIV/SIV-induced BBB disruption and neuropathogenesis in HAND.

Keywords: AIDS, Blood-brain barrier, HIV encephalitis, Netrin-1, Pericytes

\section{Background}

The blood-brain barrier (BBB) plays a crucial role in maintaining homeostasis within the brain by regulating molecules entering and exiting the brain parenchyma to prevent neural disruption $[1,2]$. The $\mathrm{BBB}$ is primarily formed by a single layer of endothelial cells, held together by tight junction proteins (TJPs) and adherent proteins, which defines the luminal space and creates

\footnotetext{
* Correspondence: kimw@evms.edu

${ }^{1}$ Department of Microbiology and Molecular Cell Biology, Eastern Virginia Medical School, 700 W. Olney Road, Lewis Hall 3174, Norfolk, VA 23501, USA Full list of author information is available at the end of the article
}

the vessel. Pericytes located abluminally to the endothelial cells surround the vessel and are subsequently surrounded by a ring of astrocytic end-feet, providing structural support and signaling capabilities to the BBB [3-5]. The spaces between the endothelial cells and astrocytic end-feet are filled with the basement membranes, which encase the pericytes and further solidify the BBB. Historically, several neurocognitive disorders, such as Alzheimer's disease (AD), multiple sclerosis (MS), and HIV-associated neurocognitive disorder (HAND), have been associated with the dysregulation of

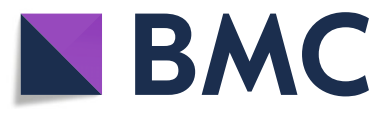

(c) The Author(s). 2019 Open Access This article is distributed under the terms of the Creative Commons Attribution 4.0 International License (http://creativecommons.org/licenses/by/4.0/), which permits unrestricted use, distribution, and reproduction in any medium, provided you give appropriate credit to the original author(s) and the source, provide a link to the Creative Commons license, and indicate if changes were made. The Creative Commons Public Domain Dedication waiver (http://creativecommons.org/publicdomain/zero/1.0/) applies to the data made available in this article, unless otherwise stated. 
the $\mathrm{BBB}$ resulting in damage to parenchymal neural structures.[6-9]

Recent research has demonstrated the relevance of the Sonic hedgehog (Shh) signaling pathway for maintaining $\mathrm{BBB}$ integrity and the loss thereof in brain aging and neurocognitive disorders [10-13]. In the adult brain, astrocytes secrete Shh. Upon secretion, Shh binds and inactivates its receptor Patched-1 (Ptch1), which results in the activation of Smoothened (Smo) and subsequent activation of Gli family transcriptional factors including Gli-1 [10, 14]. While the complete functionality of the Shh signaling pathway is not known, recent studies show that netrin-1 plays an important role in Shh-induced upregulation of TJPs, which are critical for maintaining the selective permeability of the BBB $[10,13,15,16]$.

Despite advances in antiretroviral therapy (ART), HIV-infected patients continue to suffer from HAND, which have been commonly linked to the breakdown of the BBB [17-19]. Previous studies clearly demonstrate the advantages of using a simian immunodeficiency virus (SIV) macaque model to imitate BBB breakdown in the brains of HIV-infected patients [20, 21]. Using this model, we studied changes in Shh signaling and neurovascular supporting cells in the brains of uninfected adult macaques (UI), SIV-infected macaques with no signs of encephalitis (SIVnoE) or with encephalitis (SIVE). A better understanding of the effects of HIV/SIV on the Shh pathway and periendothelial support structures at the glio-vascular interface will help identify potential therapeutic targets to reduce BBB breakdown in HIV-infected patients.

\section{Methods}

\section{Animal cohorts}

A total of 14 adult male rhesus macaques (Macaca mulatta) were used in this study. All procedures of this study were approved by the Tulane University Institutional Animal Care and Use Committee (IACUC), and were carried out in accordance with the National Institutes of Health "Guide for the Care and Use of Laboratory Animals", the recommendations of the Weatherall report, "The use of non-human primates in research," and the ARRIVE (Animal Research: Reporting In Vivo Experiments) guidelines. All of the animals were housed at the Tulane National Primate Research Center (TNPRC) in accordance with Tulane University's IACUC regulations and TNPRC endpoint policies. Endpoint policies define 15\% weight loss within 2 weeks, unresponsive opportunistic infection, persistent anorexia, severe intractable diarrhea, progressive neurological signs, significant cardiac and/or pulmonary signs, or any other serious illness as adequate terms for euthanasia. This study was conducted on brain tissues from four uninfected, four SIVnoE, and six SIVE animals (Additional file 1: Table S2). Infection groups were intravenously infected with SIVmac251 or SIV0302-2, and all the animals were not perfused at necropsy. Formalin-fixed, paraffin-embedded sections of archival brain tissues from the temporal and occipital cortices were sliced at 5-um thickness. SIVE status was determined by the presence of SIV Gag proteins in the brain, accumulation of macrophages, and the presence of multi-nucleated giant cells (MNGCs).

\section{Human brain tissues}

Formalin-fixed, paraffin-embedded sections of temporal and occipital cortices were obtained from the Manhattan HIV Brain Bank, a member of the National NeuroAIDS Tissue Consortium. A total of 4 HIVE cases with 4 seronegative controls that had been previously described elsewhere were examined $[22,23]$.

\section{Immunofluorescence microscopy}

Immunofluorescence (IF) microscopy was performed using the primary antibodies listed in Supplementary Table 2. The specificity of primary antibodies was checked in negative controls that omit the primary antibody only. Briefly, sections were incubated at $60^{\circ} \mathrm{C}$ overnight before being de-paraffinized and rehydrated in serial xylene and ethanol baths. Pretreatment with Trisor citrate-based Antigen Unmasking Solution (Vector Laboratories, Burlingame, CA) was done in a microwave $(1000 \mathrm{~W})$ for $20 \mathrm{~min}$. After cooling for $20 \mathrm{~min}$, slides were then washed in phosphate-buffered saline (PBS) containing $0.2 \%$ fish skin gelatin (FSG) (PBS/FSG). Permeabilization was achieved through incubation with PBS/FSG and 0.1\% Triton X-100 at room temperature for $1 \mathrm{~h}$ before slides were washed in PBS/FSG. Sections were then blocked with either $5 \%$ horse or goat serum for 30 min before the first primary antibody, diluted with PBS/FSG, and was applied at room temperature for $1 \mathrm{~h}$. After PBS/FSG baths, secondary antibodies conjugated to Alexa Fluor 488 or 594 (Molecular Probes, Eugene, OR) were diluted at 1:500 in PBS/FSG and applied for 1 $\mathrm{h}$ at room temperature. Subsequent primary and secondary antibodies were applied as described above. Some sections were then counterstained with DAPI for $5 \mathrm{~min}$. After IF staining was complete, sections were washed before being soaked in a quenching solution of $10 \mathrm{mM}$ $\mathrm{CuSO}_{4}$ for $45 \mathrm{~min}$. The sections were then washed with distilled water and mounted using a coverslip and Aqua-Mount aqueous mounting medium (Thermo Scientific, Waltham, MA).

A VectaFluor Excel DyLight kit (Vector) was used for Zona Occludin 1 (ZO1) IF staining. All steps described above were followed for this staining, except that horse serum was left on for $1 \mathrm{~h}$ instead of $30 \mathrm{~min}$, and the kit's Amplifier and Reagent were used for 15 and $30 \mathrm{~min}$ respectively instead of an Alexa Fluor secondary antibody. 
Fluorescent microscopy images were taken with a Zeiss Axio Observer. Z1 fluorescent microscope with a $\times 20$ or $\times 40$ objective. Zeiss AxioVision 4.9.1 edition was used to capture and merge images. Confocal images were taken with a Zeiss 880 Laser scanning confocal microscope with a $100 \times$ emersion oil objective. ZenBlack and ZenBlue programs were used to capture and merge images.

\section{Quantitative analysis}

Quantitative analysis was performed using Image J and ZenBlack. Intensity density per field was determined through thresholding images to isolate positive immunoreactivity (IR) and using the measure tool to determine the intensity density. Thresholding was achieved by locating areas of each image without specific immunoreactivity, measuring the man pixel intensity (MPI), and averaging the MPI of all images. The average MPI for non-specific staining in the images was then used as a lower limit threshold to subtract background/auto-fluorescence. MPI was determined by thresholding images to isolate positive IR and using the measure tool to find the average intensity of the selected pixels. Percent area was determined by thresholding to obtain only positive IR and using the measure tool to determine what percent of the frame contains positive staining. Total intensity was obtained by adding the pixel intensity of each pixel within the selected area into a raw score demonstrating the IR in that area. The percent of vessels demonstrating fibrinogen extravasation was determined by running linear plot profiles on the green and red channels of individual vessels captured at $\times 400$ magnification and graphing them in GraphPad as dual overlay histograms. The histograms were then analyzed to determine whether the fibrinogen was above background levels outside of the two primary glucose transporter 1 (GLUT1) peaks; vessels that displayed this phenotype were considered to be extravasated. The number of extravasated vessels was divided by the total number of vessels counted to calculate the percentage of vessels extravasated. Confocal Z-stack images were analyzed through a ZenBlack co-localization experiment. Thresholds for PDGFRB and SIVp28 were set to isolate positive IF, and the program determined the degree of co-localization between the channels. Finally, each SIVE animal was given a pathological disease score based upon the average number of lesions per centimeter square of white matter determined by observation of 15 sections per animal.

All quantifications were originally carried out in gray and white matter separately; however, while some differences in the amount of IR were seen between the two tissue types, there were no significant differences between infection groups. As such, gray and white matter were combined to form a single data point for each animal. Additionally, no lesion-associated vessels were taken into consideration during quantification unless specifically stated as such.

Pericyte thickness was calculated by measuring the total area of the pericyte coverage, excluding endothelial and luminal areas, and dividing the pericyte area by vessel area. This calculation allows for the measurement of pericyte thickness which is normalized to exclude variation caused by the size of vessels. Pericytes were determined to be hypertrophied when they were greater than $150 \%$ luminal area by a receiver operating characteristic curve analysis.

A non-lesion-associated, normal-appearing small vessel is defined as a vessel less than $15 \mu \mathrm{m}$ in luminal diameter showing endothelial continuity with no signs of extra-luminal thrombosis or perivascular accumulation of white blood cells. In the interest of ensuring that all vessels were nearly horizontal cross-sections, no single luminal radius was permitted to be more than twice the length of the shortest luminal radius. Lesions were identified through both SIVp28+ staining and accumulation of no less than eight CD68+ macrophages. All lesions in images are circled with dotted lines.

\section{Statistical analysis}

GraphPad Prism 7.2 was used to graph data and analyze its significance. A one-way ANOVA in conjunction with a Dunn's multiple comparison test was performed to determine the significance of numerical comparisons between study groups. A two-tailed paired $t$ test was used to determine significant differences between types of vessels within the same animal. * denotes $p<0.05$, * denotes $p<$ 0.01 , $*$ denotes $p<0.001$, $* * *$ denotes $p<0.0001$. All error bars denote standard deviation (SD).

\section{Results}

\section{Shh expression during SIV infection}

In this study, we sought to investigate changes in the Shh pathway at the BBB following SIV infection. First, we examined Shh protein expression in the brains of uninfected, SIVnoE and SIVE macaques by semi-quantitative IF microscopy (Supplementary Table 1). Cells within the glio-vascular interface that demonstrated an association with Shh IR in uninfected macaques were identified as astrocytes and endothelial cells via triple immunofluorescence staining for astrocyte marker glial fibrillary acidic protein (GFAP), endothelial cell marker GLUT1, and Shh (Fig. 1). In addition to neurons, these cells are known to be important contributors to the Shh pathway as astrocytes are considered a primary cellular source of Shh, and endothelial cells act as its primary site of action [10]. Shh IR was found in astrocytic processes and end-feet, and in close proximity to the endothelium (Fig. 1). 

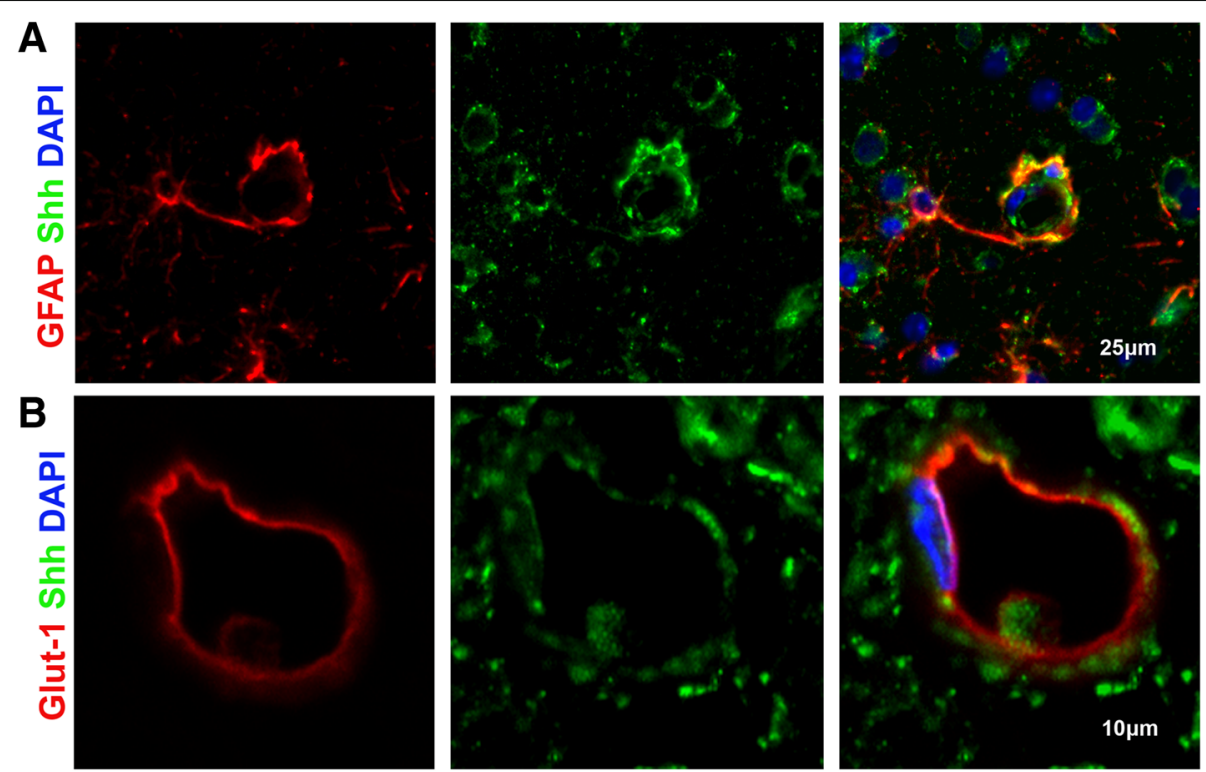

Fig. 1 Shh found on astrocytic end-feet and near endothelial cells within the glio-vascular unit triple-label IF imaging of uninfected animals for GFAP (red), Shh (green), and DAPI (blue) confirmed the presence of Shh on astrocytic end-feet (a). Likewise, IF imaging of uninfected animals for GLUT1 (red), Shh (green), and DAPI (blue) showed Shh located adjacent to endothelial cells $\mathbf{b}$

Overall, Shh IR did not differ significantly between the three groups (Fig. 2a) either for white or gray matter (data not shown). As there was no statistically significant difference in white and gray matter between the groups, no separate analyses of the gray matter and the white matter were made. Since astrogliosis was observed in all SIV-infected macaques (Additional file 1: Table S1), we initially expected an increase in Shh IR in astrocytes. However, enhanced Shh was only noted in proximity to the endothelium of SIV-infected monkeys (Fig. 2b). Interestingly, Shh levels remained high adjacent to lesion-associated vessels in SIVE animals suggesting its continued availability during lesion formation (Fig. 2c, d). Shh IR persists during SIV infection and SIVE lesion formation, suggesting any breakdown in the signaling cascade is not likely due to lack of available Shh proteins (Fig. 2).

\section{Dysregulation of netrin-1 and PDGFRB during SIV infection}

Considering the persistent presence of Shh at the BBB after SIV infection, we investigated whether downstream effectors of the pathway were subject to dysregulation. Netrin-1 is known as an important downstream effector of the Shh signaling cascade and best known for its role in promoting TJPs and maintaining the stability of the $\mathrm{BBB}[13,24]$. Building upon published literature that strongly suggests the presence of netrin-1 in endothelial cells [13], we performed triple-label immunofluorescence imaging for GLUT1 and netrin-1 in uninfected macaque cortical brain tissue (Fig. 3a). To our surprise, there was no co-presence of netrin-1 and GLUT1 IR. However, subsequent staining for PDGFRB and netrin-1 showed co-localization, indicating pericytes as the major resident cellular source of netrin-1 at the BBB (Fig. 3b). Further investigation of netrin-1 showed a significant increase in netrin-1 MPI in SIVE animals when compared to the uninfected (Fig. 4a). In support of the idea that netrin-1 downstream signaling is maintained after infection, we found no significant difference in ZO1 IR associated with normal-appearing small vessels (see "Methods" section for definition) across groups (Additional file 1: Figure S1b). Interestingly, these findings were accompanied by the observation that pericyte thickness was significantly increased in SIVE animals when compared to the uninfected (Fig. 4b). Since both pericyte thickness and netrin-1 IR were increased in SIVE animals and ZO1 IR was maintained, we initially expected to find the continued presence of pericytes in lesion-associated vessels. However, neither netrin-1 nor PDGFRB IR was found around lesion-associated vessels (Fig. 4c, d), nor was there detectable IR for ZO1 (data not shown). The absence of pericytes in the lesion was further confirmed using a second pericyte marker CD146, which was present around non-lesion-associated vessels, but lacking in lesions (Figure S1e). While netrin-1 IR and pericyte thickness are increased during SIV infection with the development of SIVE, both are absent in lesions suggesting potential disruption of the 


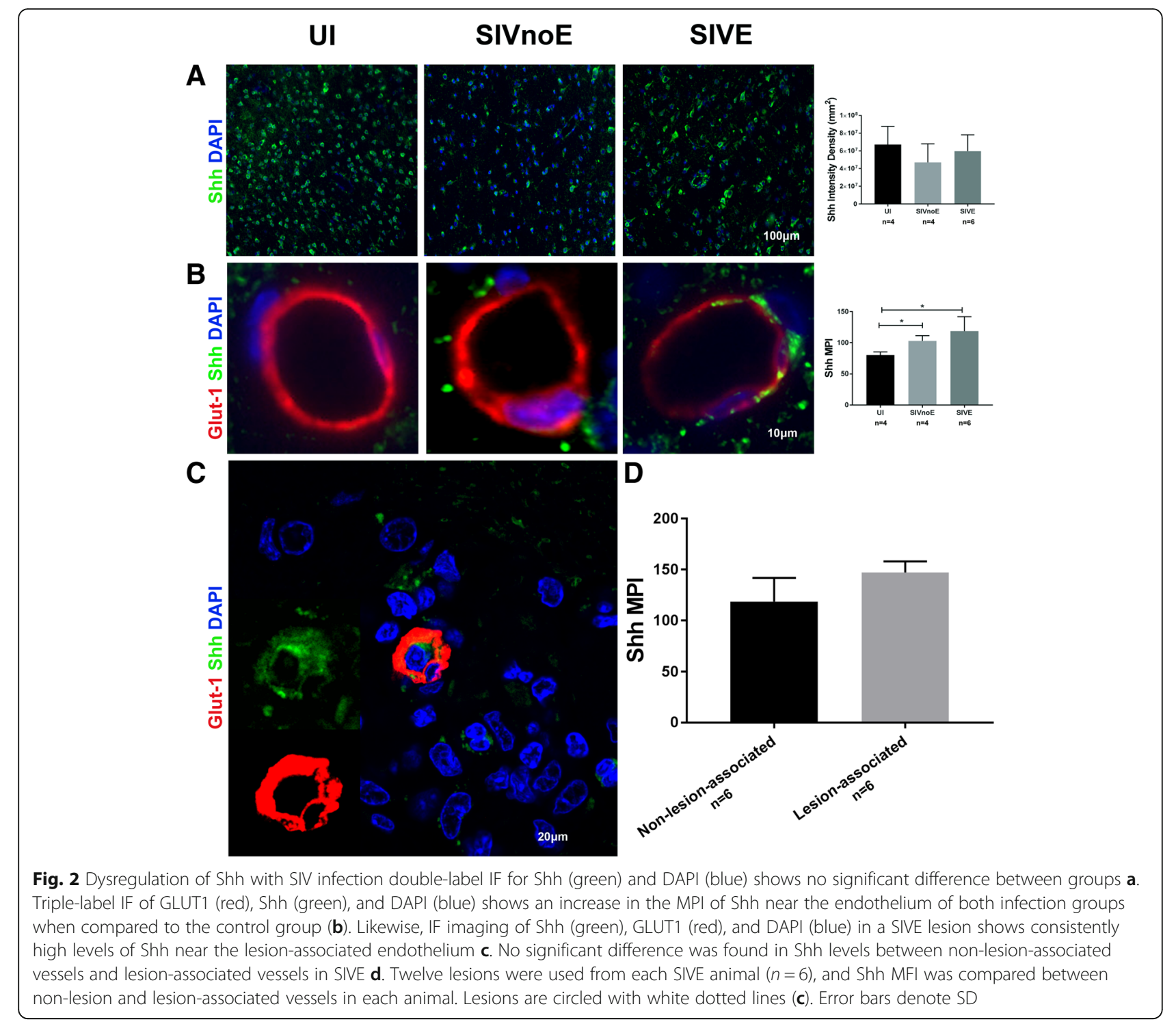

Shh signaling pathway in relation to lesion formation (Fig. 4).

\section{Increase in pericyte thickness due to hypertrophy, not proliferation}

To help clarify this morphological change of pericytes after SIV infection, we first investigated whether the increase in pericyte coverage around the endothelium was due to an increase in the number of pericytes surrounding these vessels or the size of each pericyte. By plotting the number of pericytes around each vessel against the thickness of pericyte coverage, we were able to determine that there was no significant correlation between the two (Fig. 5a). This, in conjunction with our finding that total PDGFRB IR did not change between study groups (Fig. 5b) despite the observed change in pericyte thickness (Fig. 4b), lead us to the conclusion that pericyte size, not number, was responsible for pericyte thickening. This was further confirmed through confocal microscopy. High magnification images $(\times 1000)$ were taken of endothelial cell coverage by both hypertrophied and non-hypertrophied pericytes on the same section of SIVE cortical brain tissue to show the visual difference between the two (Fig. 5c, d). This data suggests that thickened pericyte coverage is likely due to damage-induced hypertrophy of the cells, not a proliferation-based response mechanism to infection (Fig. 5).

\section{Hypertrophied pericytes are associated with BBB breakdown and SIV infection}

To further evaluate the connection between pericyte hypertrophy and SIV infection, we studied the breakdown of the BBB. Fibrinogen extravasation is an 

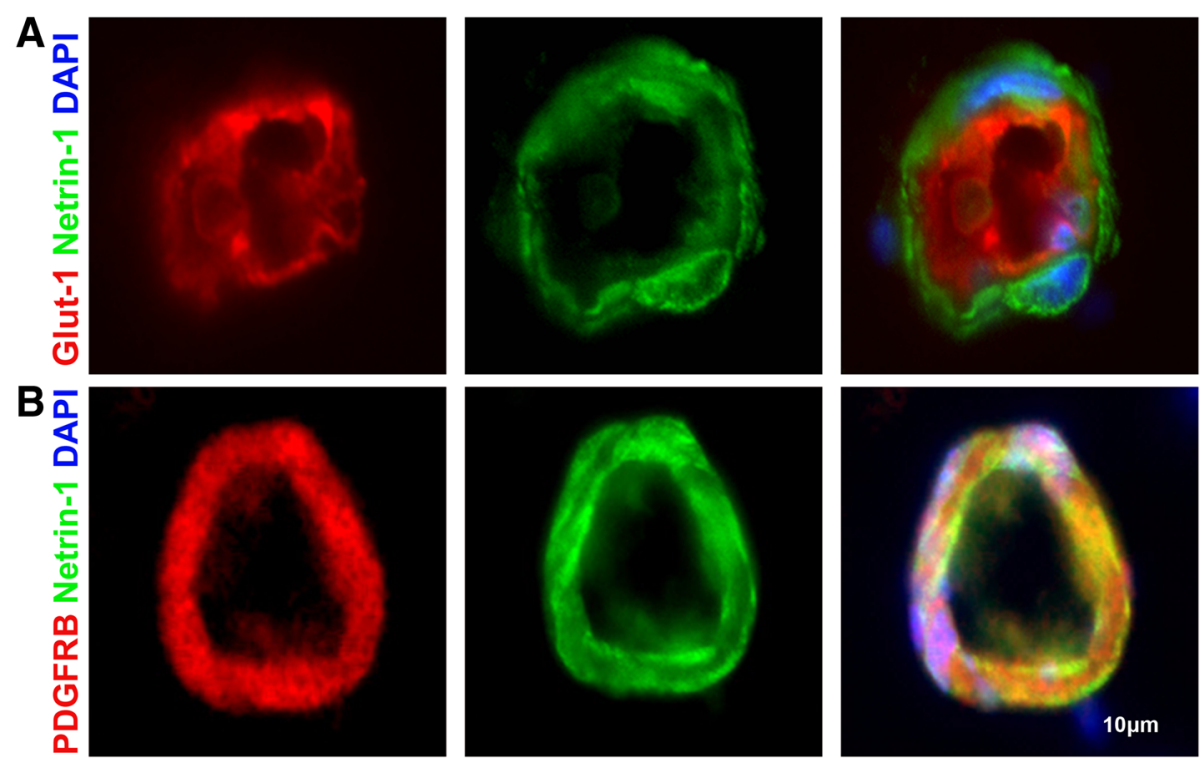

Fig. 3 Netrin-1 found in pericytes within the neurovascular niche triple IF staining of uninfected animals for GLUT1 (red), netrin-1 (green), and DAPI (blue) shows netrin-1 surrounding the endothelium (a). Triple IF staining of uninfected animals for PDGFRB (red), netrin-1 (green), and DAPI (blue) shows netrin-1 co-localizing with pericytes at the glio-vascular interface $\mathbf{b}$

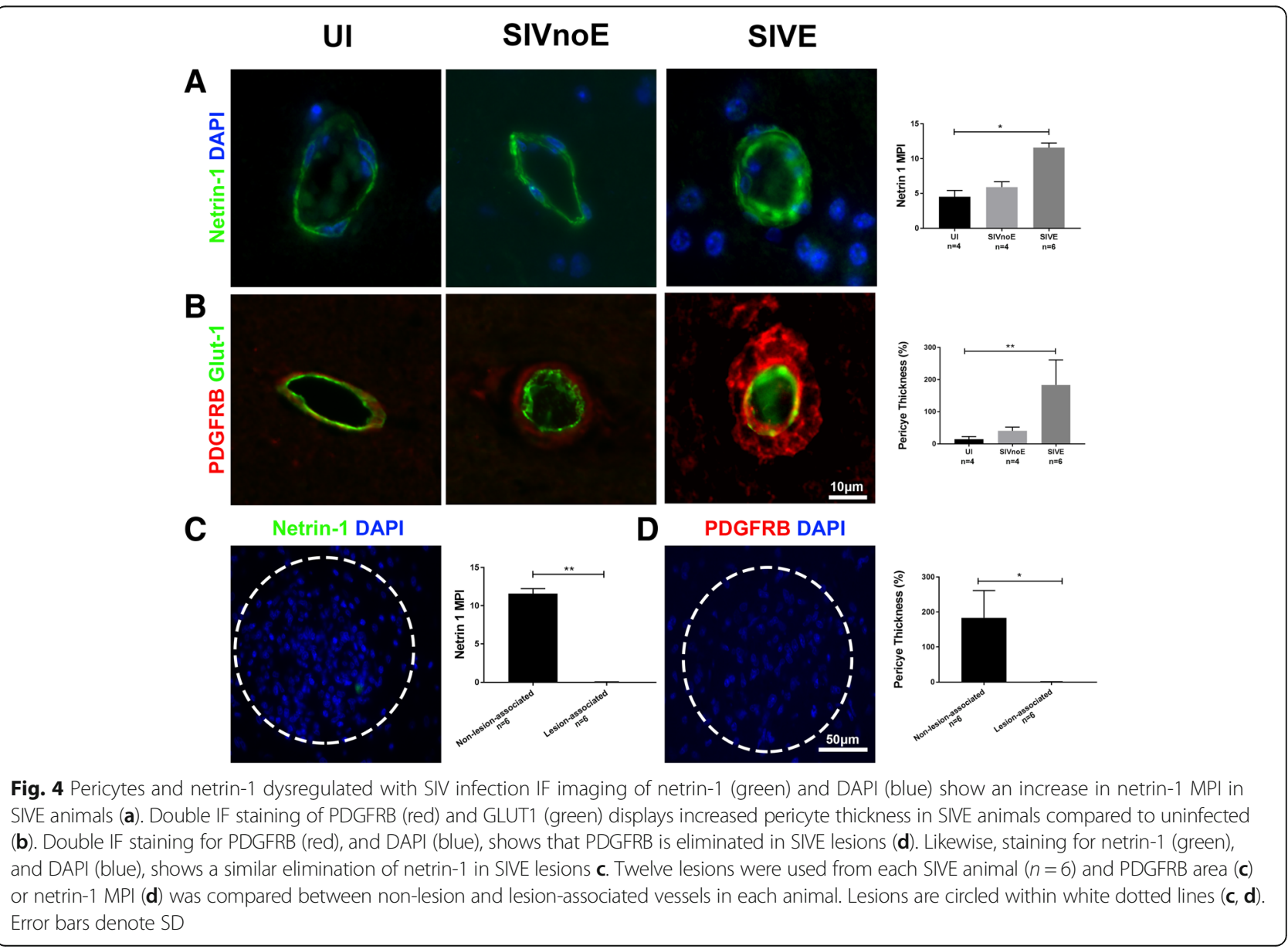



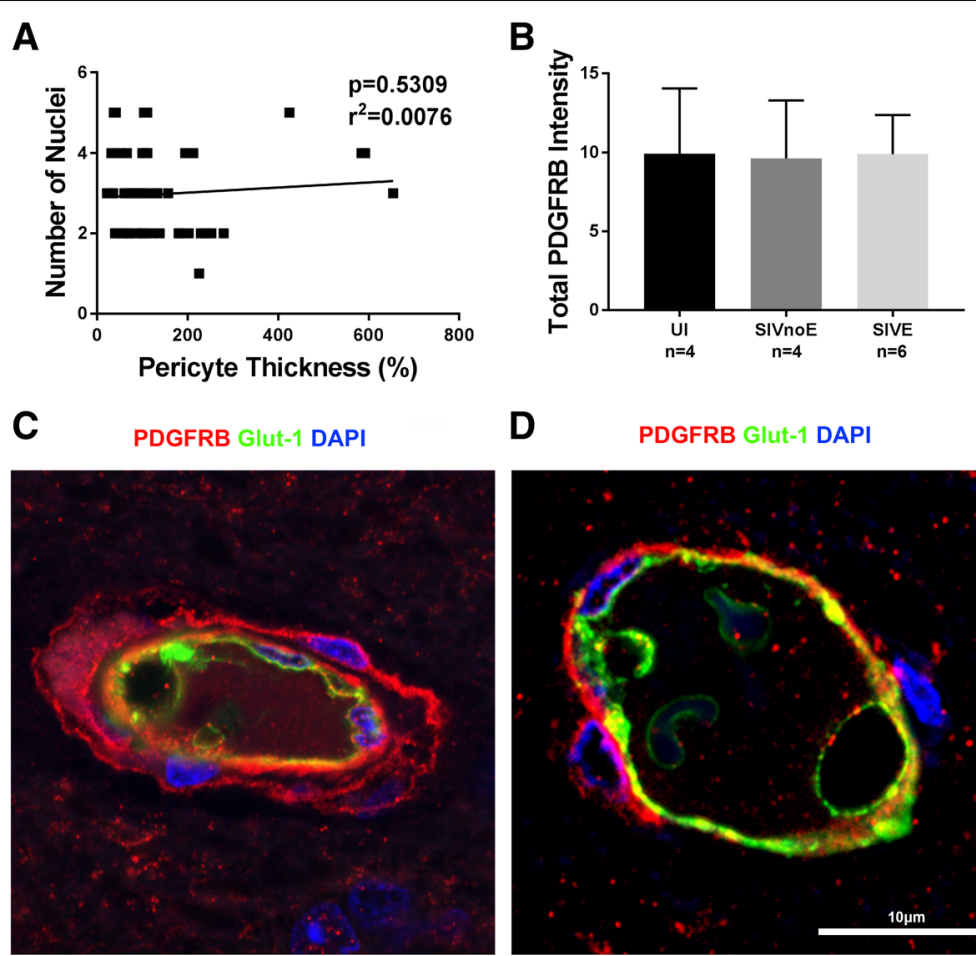

D PDGFR Glut-1 DAPI
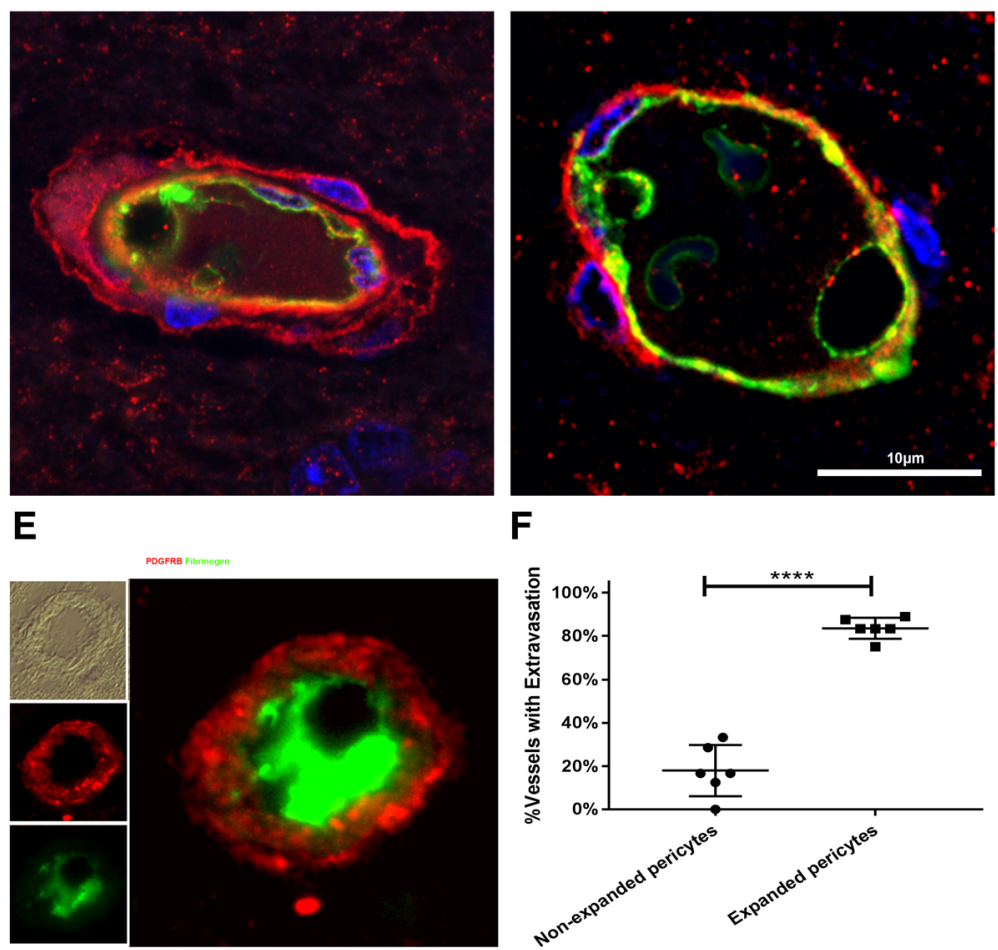

Fig. 5 Changes in pericyte thickness of SIVE brain are due to cellular changes, not pericyte number. Pericyte thickness graphed against the number of pericytic nuclei shows no significant trend between the number of pericytes and their thickness around the endothelium of SIVE infected animals (a). Total PDGFRB intensity shows no change in PDGFRB expression between study groups despite the significant difference in pericyte area (b). Triple-label IF for PDGFRB (red), GLUT1 (green), and DAPI (blue) of an expanded pericyte (c) and a non-expanded pericyte (d) show the cellular differences between two pericytes in the same SIVE animal (10A067). Using double-label IF for PDGFRB (red) and fibrinogen (green), we were able to determine that $80 \%$ of vessels surrounded by enlarged pericytes showed signs of fibrinogen extravasation (e, f). Error bar denotes SD. Ten vessels were randomly chosen from each SIVE animal $(n=6)$; each point on the graph a denotes a single vessel. No significant difference was found in $\mathbf{a}$ or $\mathbf{b}$. A paired $t$ test was used to compare between samples in $\mathbf{f}$

accepted method of measuring BBB disruption in post-mortem samples by visualizing endogenous blood plasma proteins [25]. First, we measured the percent of vessels demonstrating fibrinogen extravasation $(n=500$ per animal) and found a significant increase in SIVE animals when compared to both uninfected and SIVnoE groups (Figure S1c). These data were further visualized by dual histogram overlays in which any fibrinogen IF (red) that occurs outside of the two primary GLUT1 IF (green) peaks on the $x$-axis is considered to be extravasated fibrinogen (Figure S1d). In addition, we found that $80 \%$ of vessels with hypertrophied pericyte coverage $(n=60)$ showed fibrinogen extravasation, while it occurred in less than $20 \%$ of vessels covered by non-hypertrophied pericytes $(n=60)$ (Fig. 5e, f). This demonstrates an association between areas of BBB breakdown and hypertrophied pericyte coverage.

To determine how this may relate to SIV infection, we studied the association between hypertrophied pericytes and SIVp28 protein IR in SIVE. In examining 60 hypertrophied and 60 non-hypertrophied pericytes from SIVE animals, we found that all 60 vessels with hypertrophied 


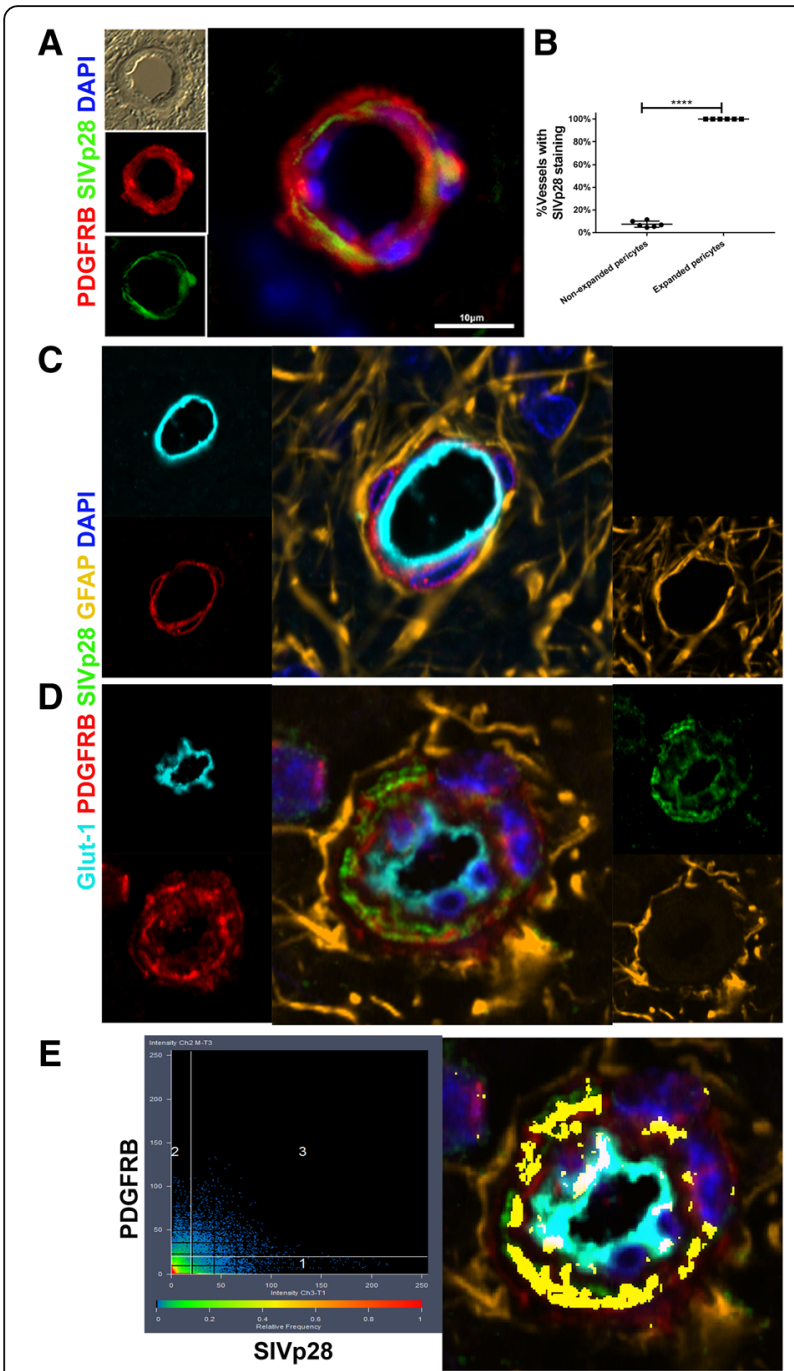

Fig. 6 Enlarged pericytes highly localized to areas of fibrinogen extravasation and pericyte infection IF imaging for PDGFRB (red), SIVp28 (green), and DAPI (blue) a of 10 vessels with and 10 without enlarged pericyte coverage for each SIVE animal $(n=6)$ showed that $100 \%$ of the enlarged pericytes $(n=60)$ had localized SIVp28+ staining, while only $8 \%$ of the non-enlarged pericytes $(n=60)$ showed positive staining (b). Five-color IF staining for GFAP (gold), PDGFRB (red), GLUT1 (aqua), SIVp28 (green), and DAPI (dark blue) show the major cellular components of the glio-vascular interface and how they localize with SIVp28 viral protein marker in the nonhypertrophied pericyte of an uninfected animal (11A014) and the hypertrophied pericyte of an SIVE animal (11A554) (c, d). This demonstrates the presence of SIVp28 primarily in the pericytes and endothelial cells (d). Presence of SIVp28+ staining within pericytes was confirmed via a confocal microscopy Z-stack of a 20-um-thick section using $0.25 \mu \mathrm{m}$ increments analyzed with a ZenBlack colocalization test in which points in quadrant 3 and highlighted in yellow are considered to be co-localized between PDGFRB and SIVp28 (e). A paired $t$ test was used to determine significance between groups in $\mathbf{b}$ pericytes showed SIVp28 localization to the pericytes, while only $8 \%$ of non-hypertrophied pericytes demonstrated SIVp28 localization (Fig. 6a, b). To determine whether hypertrophied pericytes contained SIV Gag p28, we utilized a five-color immunofluorescence staining to visualize the major cell components within the glio-vascular unit. A side-by-side comparison of a non-hypertrophied vessel from an uninfected animal and a hypertrophied vessel from a SIVE animal shows marked differences between the vessel phenotypes particularly in pericyte organization (Fig. 6c, d). A ZenBlack co-localization test of confocal Z-stack images of $20-\mu \mathrm{m}$-thick sections displaying astrocytes, pericytes, endothelial cells, their nuclei, and SIVp28 protein at the $\mathrm{BBB}$ showed that these hypertrophied pericytes were positive for SIV Gag p28 (Fig. 6e). In support of this, in vitro studies showing the capability of HIV-1 to enter and replicate within human brain pericytes suggest that HIV-1 is capable of infecting these cells [26, 27].

\section{Shh persistence and infection of hypertrophied pericytes confirmed in human cortical brain tissue}

To confirm that our findings in rhesus macaques hold clinical relevance for humans infected with HIV, we examined four uninfected and four HIVE cases. Like with the animal model, there was a significant difference in the amount of Shh localized to the endothelium between uninfected and HIVE groups, which persisted within lesion-associated vessels (Fig. 7a, c). Additionally, we found a significant increase in pericyte thickness in subjects with HIVE compared to uninfected, but again, no signs of PDGFRB IR around lesion-associated vessels (Fig. 7b, d). Z-stacking was performed to demonstrate the presence of HIV p24 antigen within hypertrophied pericytes in the brains of HIVE patients (Fig. 7e). In support of our earlier findings in SIV-infected rhesus macaques, this result demonstrates that pericytes containing HIVp24 become hypertrophied in the glio-vascular unit of HIVE patients despite consistent availability of the Shh protein.

\section{Disease severity is strongly correlated with dysregulation} of ZO1, pericyte morphology, and BBB permeability

To determine whether disease progression played a role in the severity of changes seen, we calculated a disease score for each SIVE animal by calculating the number of lesions per centimeter square of white matter. We found that ZO1 expression is negatively correlated with the severity of SIVE (Fig. 8a). Pericyte thickness and fibrinogen extravasation are both strongly positively correlated with the severity of SIVE, suggesting that hypertrophied pericytes and increased frequency of BBB disruption may be associated with SIVE progression in SIV-infected macaques (Fig. 8b, c). Our model suggests that hypertrophied pericytes may be involved in the initiation of $\mathrm{BBB}$ 


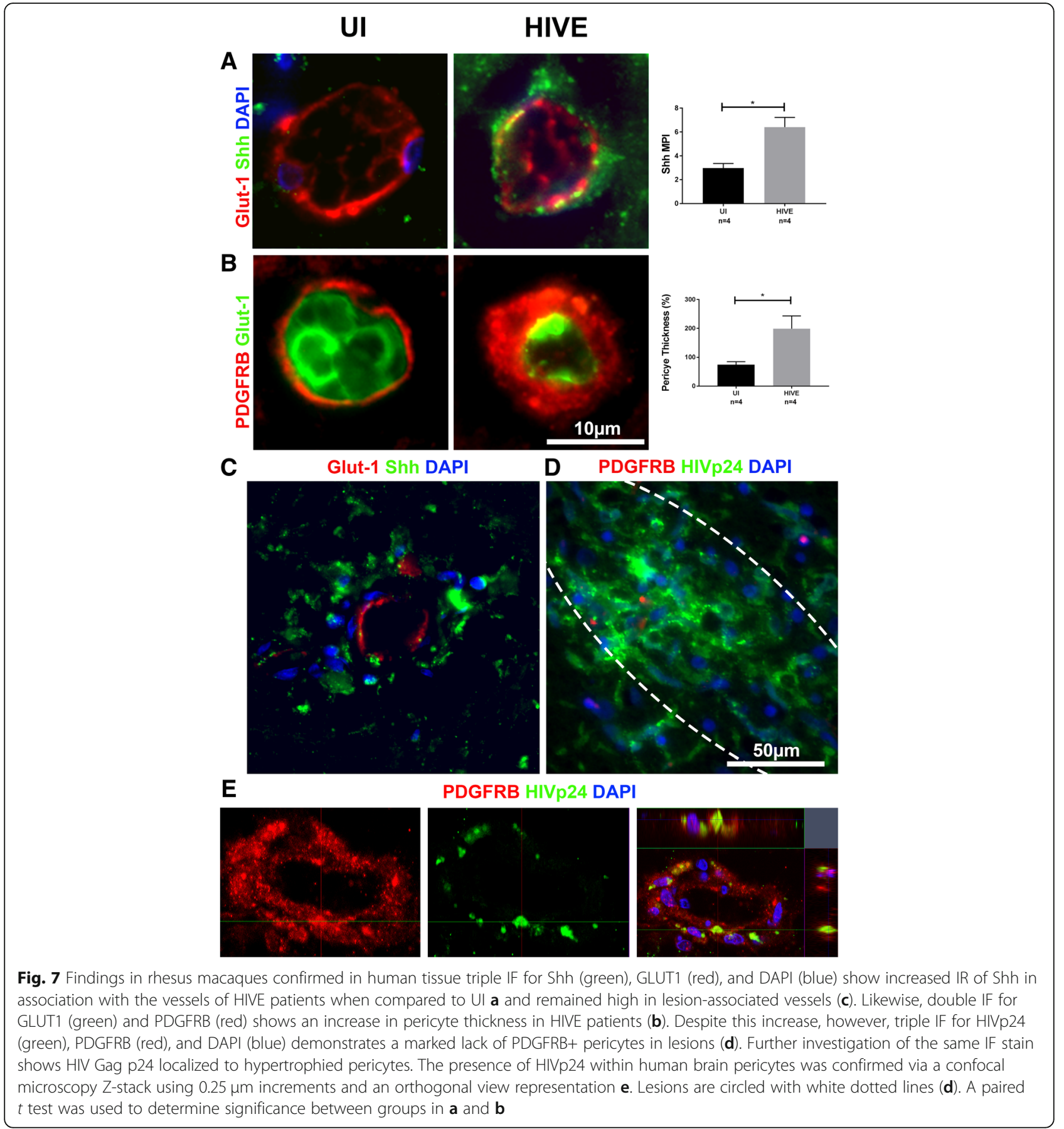

destabilization in SIV-infected animals, and the loss of pericytes further disrupts the BBB stability (Fig. 8d).

\section{Discussion}

A recent study in a humanized mouse model suggests a decrease in Shh production with HIV infection [28]. However, our study found no significant changes in overall Shh production and instead an increase in Shh localized to the endothelium within the lesion and to non-lesion-associated vessels in SIV-infected rhesus macaques. The use of different models and stages of infection may account for this difference. We also noted the absence of PDGFRB+ pericytes and netrin-1 within HIVE/SIVE lesions suggesting that the loss of pericytes may play a role in HIV/SIV-induced BBB breakdown and lesion formation. Our findings may help to direct future therapeutic studies surrounding pericyte damage and loss in HIV/SIV infection and underline the 


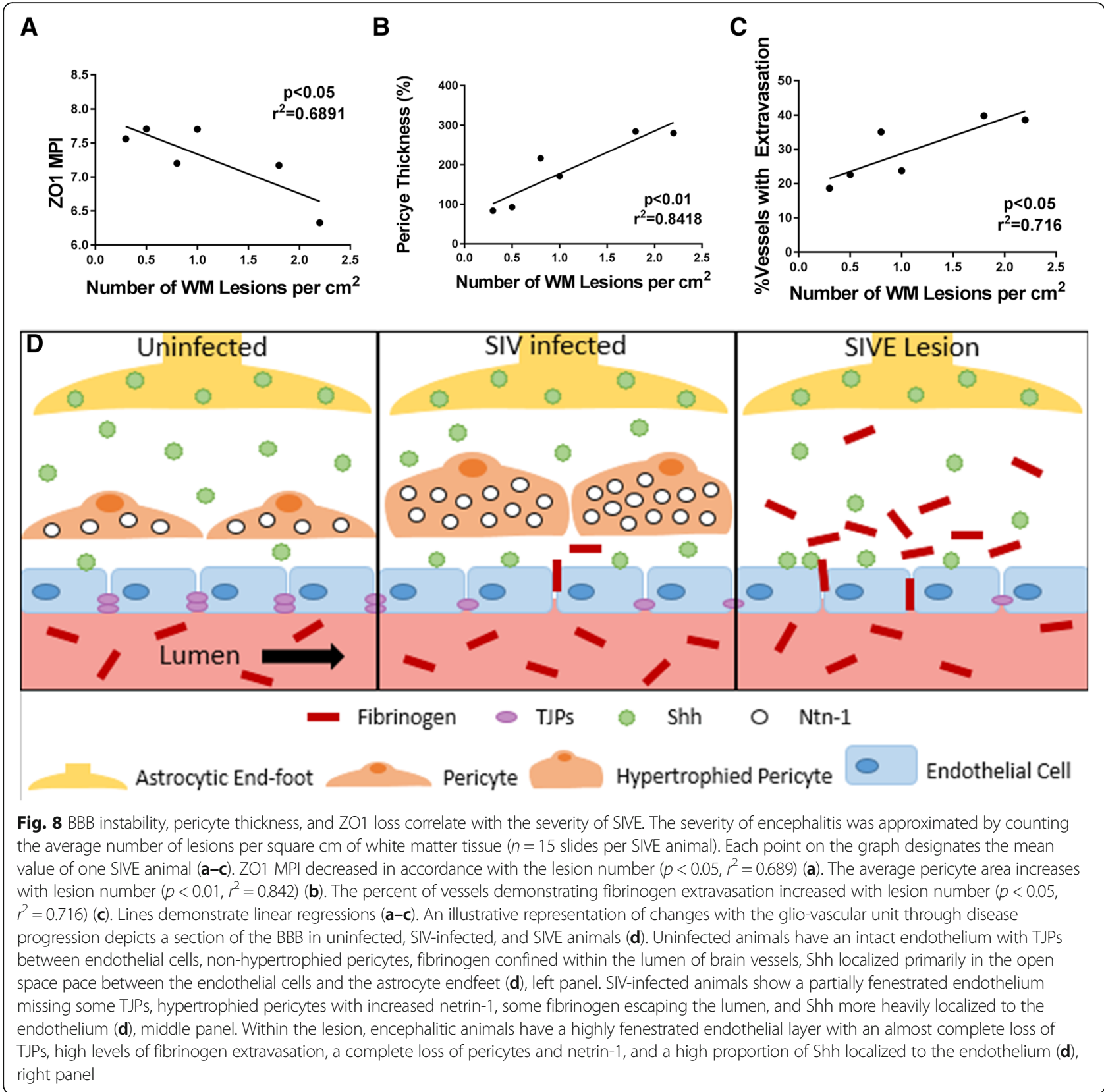

importance of pericyte preservation and Shh downstream effector stability in maintaining the selective permeability of the BBB during HIV/SIV infection.

Shh has been noted as a significant factor in damage repair across multiple neurocognitive diseases and disorders including MS and ischemic brain injury, as well as HAND. Shh has been seen in astrocytes in all stages of MS lesions as well as in normal appearing white matter within the MS brain, but high levels of Shh + macrophages tend to be present in demyelinating lesions and less so in remyelinating lesions, which primarily house Shh + axons $[10,29,30]$. A similar increase in Shh is found within areas of ischemic stroke, and an inhibition of Smo has been shown to prevent natural recovery, while eliminating Shh has been shown to reduce the number of regionally produced Olig $2+$ cells during the post-ischemia recuperation process [11, 31-33]. These studies and many others show the reparative abilities of Shh within the glio-vascular unit, but recent studies have suggested that HIV is capable of disrupting the production of Shh preventing its continued maintenance and repair of the BBB [28]. Despite the presence of Shh in the brain of SIV-infected macaques, the loss of netrin-1 within SIVE lesions suggests that a downstream disruption of the Shh pathway may play a role in BBB breakdown. Evidence suggests that the loss of netrin-1 is 
likely due to the loss of its host cell, the pericyte, which become hypertrophied in association with HIV/SIV infection of the brain, and is absent in encephalitic lesions.

Pericyte dysfunction is not unique to HIV/SIV infection. Previous studies suggest that pericyte dysfunction may play a key role in diseases like $\mathrm{AD}$, cancer, seizure disorders, and even natural aging. Pericytes have been a key target in $\mathrm{AD}$ research due to their ability to traffic amyloid beta deposits in the brain back into the bloodstream for removal by other organs, making their dysfunction likely to result in AD-like brain pathology [3439]. Recently, high numbers of cells expressing NG2, a commonly used pericyte marker, have been seen within high-grade choroid plexus tumors suggesting a potential role for pericytes in cancer [40]. Pericytes have also been suggested to be involved with the cerebrovascular rearrangement seen in seizure patients [41]. Changes in pericytes during normal aging have been linked to localized hypoxia as well as BBB leakage and an increase in neurotoxicity within the brain [42]. Studies with animal models of traumatic brain injury suggest that pericytes play a key role in the construction and repair of the brain's microvascular structures [43, 44]. Although studies investigating the effects of HIV/SIV on brain pericytes reported an overall decrease in pericyte coverage [45-47], we found that pericytes around many of the vessels in SIVE animals are becoming hypertrophied. This suggests that they may be undergoing functional changes or damage prior to being eliminated upon lesion formation. While no ART-treated animals were investigated in this study, Fig. 8 shows that both pericyte thickness and fibrinogen extravasation are positively correlated with the severity of SIVE suggesting that pericyte hypertrophy may continue to contribute to BBB disruption even in virally suppressed individuals.

Further research is needed to fully understand changes in the Shh pathway in the adult brain during HIV/SIV infection and the effect on the BBB. Additionally, further studies are needed in order to determine the cause and effect of pericyte hypertrophy in adult macaques with SIVE. Despite suggestions concerning Shh as a potential therapeutic target for HIV-associated BBB breakdown [28], the constitutive expression and increased localization of Shh to the endothelium in vivo calls into question its value as a therapeutic agent. Disruption of downstream effectors of the Shh pathway, such as netrin-1, may still play a key role in the dysregulation of the BBB during HIV/SIV infection due to SIV-associated pericyte dysfunction, which may result in a disruption of this and other key pathways at the glio-vascular interface.

\section{Conclusions}

In conclusion, the current study provided evidence for brain pericyte hypertrophy and loss, concomitant with disruption of BBB, in the brains of SIV-infected macaques and HIV-infected patients. Further research on pericyte dysfunction with HIV/SIV infection is needed to determine its role in the dysregulation of the Shh pathway and HAND as well as to identify any links to other neurocognitive disorders and diseases.

\section{Additional files}

\begin{abstract}
Additional file 1: Table S1. Animals recruited in this study. Table S2. Primary antibodies used in study. Fig. S1. Astrocytosis and evidence of BBB breakdown observed with SIV infection Double IF staining for GFAP (red) and DAPI (blue) shows a significant increase in astrocyte coverage with SIV infection (a). Double IF staining for ZO1 (green) and DAPI (blue) shows a trending decrease in ZO1 expression with infection, but no significant difference (b). Triple-label IF of fibrinogen (red), Glut-1 (green), and DAPI (blue), shows a significant increase in the percent of vessels showing fibrinogen extravasation in SIVE animals when compared to uninfected (c). Linear analysis of MFI for both GLUT1 (green) and fibrinogen (red) provides an overlaid histogram view of the vessels in Figure S1C (d). Fibrinogen (red) that occurs outside the $x$-axis bounds of the two main GLUT1 (green) peaks is considered to be extravasated fibrinogen (d). Error bars indicate SD. Triple IF staining for vascular GLUT1 (green), pericyte CD146 (red) and nuclear DAPI (blue) shows a non-lesion-associated vessel (left) with pericyte coverage and a lesionassociated vessel (right) without pericyte coverage (e). (Docx 294 kb)
\end{abstract}

\section{Abbreviations}

AD: Alzheimer's disease; ART: Antiretroviral therapy; BBB: Blood-brain barrier; CNS: Central nervous system; FSG: Fish skin gelatin; GFAP: Glial fibrillary acidic protein; GLUT1: Glucose transporter 1; HAND: HIV-associated neurocognitive disorders; IACUC: Institutional Animal Care and Use Committee;

IF: Immunofluorescence; IR: Immunoreactivity; MNGC: Multinucleated giant cell; MPI: Mean pixel intensity; MS: Multiple sclerosis; PBS: Phosphate-buffered saline; PDGFRB: Platelet-derived growth factor receptor beta; Ptch1: Patched

1; Shh: Sonic hedgehog; SIV: Simian immunodeficiency virus; SIVE

SIV: Encephalitis; SIVnoE: SIV-infected without encephalitis;

Smo: Smoothened; TJP: Tight junction protein; TNPRC: Tulane National

Primate Research Center; UI: Uninfected; ZO1: Zona occludens 1

\section{Acknowledgements}

We thank Dr. Susan Morgello, of the Manhattan HIV Brain Bank (R24MH59724), for facilitating access to HIVE brain autopsy samples. We also thank Drs. Aurora Kerscher and Jerry Nadler for access to their fluorescence microscopes.

\section{Ethics approvals and consent to participate}

This article does not contain any studies with human participants performed by any of the authors. A Not Human Subjects Research determination was made by the Eastern Virginia Medical School Institutional Review Board. All applicable international, national, and/or institutional guidelines for the care and use of animals were followed. For the detailed ethical statement, please refer to Methods.

\section{Funding}

This work was supported by NIH Grants R01MH107333 and R21MH108458 (W.-K.K.); also supported in part by R01Al097059 and R33Al110163 (M.J.K).

\section{Availability of data and materials}

The datasets used and/or analyzed during the current study are available from the corresponding author on reasonable request.

\section{Authors' contributions}

DGB and AK performed the experiments, acquired and analyzed the data, and wrote the manuscript.ARF performed the experiments and helped in data analysis. MJK directed the animal studies. WKK conceived the study, directed the animal studies, analyzed the data, and wrote the manuscript. All authors read and approved the final manuscript. 


\section{Consent for publication}

Not applicable.

\section{Competing interests}

The authors declare that they have no competing interests.

\section{Publisher's Note}

Springer Nature remains neutral with regard to jurisdictional claims in published maps and institutional affiliations.

\section{Author details}

'Department of Microbiology and Molecular Cell Biology, Eastern Virginia Medical School, 700 W. Olney Road, Lewis Hall 3174, Norfolk, VA 23501, USA. ${ }^{2}$ Division of Immunology, Tulane National Primate Research Center, Covington, Louisiana, USA.

\section{Received: 8 August 2018 Accepted: 25 March 2019}

\section{Published online: 13 April 2019}

\section{References}

1. Abbott NJ, Patabendige AA, Dolman DE, Yusof SR, Begley DJ. Structure and function of the blood-brain barrier. Neurobiol Dis. 2010;37:13-25.

2. Daneman R, Prat A. The blood-brain barrier. Cold Spring Harb Perspect Biol. 2015;7:a020412.

3. Armulik A, Genove G, Mae M, Nisancioglu MH, Wallgard E, Niaudet C, et al. Pericytes regulate the blood-brain barrier. Nature. 2010;468:557-61.

4. Krueger M, Bechmann I. CNS pericytes: concepts, misconceptions, and a way out. Glia. 2010;58:1-10

5. Zhao Z, Nelson AR, Betsholtz C, Zlokovic BV. Establishment and dysfunction of the blood-brain barrier. Cell. 2015:163:1064-78.

6. Persidsky Y, Ramirez SH, Haorah J, Kanmogne GD. Blood-brain barrier: structural components and function under physiologic and pathologic conditions. J Neurolmmune Pharmacol. 2006;1:223-36.

7. Zlokovic BV. The blood-brain barrier in health and chronic neurodegenerative disorders. Neuron. 2008;57:178-201.

8. Weiss N, Miller F, Cazaubon S, Couraud PO. The blood-brain barrier in brain homeostasis and neurological diseases. Biochim Biophys Acta. 2009;1788: 842-57.

9. Spencer JI, Bell JS, DeLuca GC. Vascular pathology in multiple sclerosis: reframing pathogenesis around the blood-brain barrier. J Neurol Neurosurg Psychiatry. 2018;89:42-52.

10. Alvarez JI, Dodelet-Devillers A, Kebir H, Ifergan I, Fabre PJ, Terouz S, et al, The hedgehog pathway promotes blood-brain barrier integrity and CNS immune quiescence. Science. 2011;334:1727-31.

11. Chechneva OV, Mayrhofer F, Daugherty DJ, Krishnamurty RG, Bannerman P, Pleasure DE, et al. A smoothened receptor agonist is neuroprotective and promotes regeneration after ischemic brain injury. Cell Death Dis. 2014;5: e1481.

12. Wang $Y$, Jin $S$, Sonobe $Y$, Cheng $Y$, Horiuchi $H$, Parajuli B, et al. Interleukin1 beta induces blood-brain barrier disruption by downregulating sonic hedgehog in astrocytes. PLoS One. 2014;9:e110024.

13. Podjaski C, Alvarez Jl, Bourbonniere L, Larouche S, Terouz S, Bin JM, et al. Netrin 1 regulates blood-brain barrier function and neuroinflammation. Brain. 2015;138:1598-612

14. Osterlund T, Kogerman P. Hedgehog signalling: how to get from Smo to ci and Gli. Trends Cell Biol. 2006;16:176-80.

15. Wen J, Qian S, Yang Q, Deng L, Mo Y, Yu Y. Overexpression of netrin-1 increases the expression of tight junction-associated proteins, claudin-5, occludin, and ZO-1, following traumatic brain injury in rats. Exp Ther Med. 2014;8:881-6.

16. Xie Z, Enkhjargal B, Reis C, Huang L, Wan W, Tang J, et al. Netrin-1 preserves blood-brain barrier integrity through deleted in colorectal cancer/focal adhesion kinase/RhoA signaling pathway following subarachnoid hemorrhage in rats. J Am Heart Assoc. 2017;6:e005198.

17. Petito CK, Cash KS. Blood-brain barrier abnormalities in the acquired immunodeficiency syndrome: immunohistochemical localization of serum proteins in postmortem brain. Ann Neurol. 1992:32:658-66.

18. Dallasta LM, Pisarov LA, Esplen JE, Werley JV, Moses AV, Nelson JA, et al. Blood-brain barrier tight junction disruption in human immunodeficiency virus-1 encephalitis. Am J Pathol. 1999;155:1915-27.
19. Ivey NS, MacLean AG, Lackner AA. Acquired immunodeficiency syndrome and the blood-brain barrier. J Neuro-Oncol. 2009:15:111-22.

20. Luabeya MK, Dallasta LM, Achim CL, Pauza CD, Hamilton RL. Blood-brain barrier disruption in simian immunodeficiency virus encephalitis. Neuropathol Appl Neurobiol. 2000;26:454-62.

21. MacLean AG, Belenchia GE, Bieniemy DN, Moroney-Rasmussen TA, Lackner AA. Simian immunodeficiency virus disrupts extended lengths of the blood--brain barrier. J Med Primatol. 2005;34:237-42.

22. Holder GE, McGary CM, Johnson EM, Zheng R, John VT, Sugimoto C, et al. Expression of the mannose receptor CD206 in HIV and SIV encephalitis: a phenotypic switch of brain perivascular macrophages with virus infection. J Neurolmmune Pharmacol. 2014;9:716-26.

23. Filipowicz AR, McGary CM, Holder GE, Lindgren AA, Johnson EM, Sugimoto $C$, et al. Proliferation of perivascular macrophages contributes to the development of encephalitic lesions in HIV-infected humans and in SIVinfected macaques. Sci Rep. 2016;6:32900

24. Yu J, Li C, Ding Q, Que J, Liu K, Wang H, et al. Netrin-1 ameliorates bloodbrain barrier impairment secondary to ischemic stroke via the activation of PI3K pathway. Front Neurosci. 2017;11:700.

25. Saunders NR, Dziegielewska KM, Mollgard K, Habgood MD. Markers for blood-brain barrier integrity: how appropriate is Evans blue in the twentyfirst century and what are the alternatives? Front Neurosci. 2015;9:385.

26. Nakagawa S, Castro V, Toborek M. Infection of human pericytes by HIV-1 disrupts the integrity of the blood-brain barrier. J Cell Mol Med. 2012;16: 2950-7.

27. Cho HJ, Kuo AM, Bertrand L, Toborek M. HIV alters gap junction-mediated intercellular communication in human brain pericytes. Front Mol Neurosci. 2017; 10:410.

28. Singh VB, Singh MV, Gorantla S, Poluektova LY, Maggirwar SB. Smoothened agonist reduces human immunodeficiency virus type-1-induced blood-brain barrier breakdown in humanized mice. Sci Rep. 2016;6:26876.

29. Seifert T, Bauer J, Weissert R, Fazekas F, Storch MK. Differential expression of sonic hedgehog immunoreactivity during lesion evolution in autoimmune encephalomyelitis. J Neuropathol Exp Neurol. 2005;64:404-11.

30. Wang Y, Imitola J, Rasmussen S, O'Connor KC, Khoury SJ. Paradoxical dysregulation of the neural stem cell pathway sonic hedgehog-Gli1 in autoimmune encephalomyelitis and multiple sclerosis. Ann Neurol. 2008;64: 417-27.

31. Amankulor NM, Hambardzumyan D, Pyonteck SM, Becher OJ, Joyce JA, Holland EC. Sonic hedgehog pathway activation is induced by acute brain injury and regulated by injury-related inflammation. J Neurosci. 2009;29: 10299-308.

32. Chechneva OV, Deng W. Empowering sonic hedgehog to rescue brain cells after ischemic stroke. Neural Regen Res. 2015;10:360-2.

33. Jin Y, Raviv N, Barnett A, Bambakidis NC, Filichia E, Luo Y. The shh signaling pathway is upregulated in multiple cell types in cortical ischemia and influences the outcome of stroke in an animal model. PLoS One. 2015:10: e0124657.

34. Sagare AP, Bell RD, Zhao Z, Ma Q, Winkler EA, Ramanathan A, et al. Pericyte loss influences Alzheimer-like neurodegeneration in mice. Nat Commun. 2013:4:2932.

35. Sengillo JD, Winkler EA, Walker CT, Sullivan JS, Johnson M, Zlokovic BV. Deficiency in mural vascular cells coincides with blood-brain barrier disruption in Alzheimer's disease. Brain Pathol. 2013;23:303-10.

36. Janota CS, Brites D, Lemere CA, Brito MA. Glio-vascular changes during ageing in wild-type and Alzheimer's disease-like APP/PS1 mice. Brain Res. 2015;1620:153-68.

37. Giannoni P, Arango-Lievano M, Neves ID, Rousset MC, Baranger K, Rivera S, et al. Cerebrovascular pathology during the progression of experimental Alzheimer's disease. Neurobiol Dis. 2016;88:107-17.

38. Halliday MR, Rege SV, Ma Q, Zhao Z, Miller CA, Winkler EA, et al. Accelerated pericyte degeneration and blood-brain barrier breakdown in apolipoprotein E4 carriers with Alzheimer's disease. J Cereb Blood Flow Metab. 2016;36: 216-27

39. Miners JS, Schulz I, Love S. Differing associations between Abeta accumulation, hypoperfusion, blood-brain barrier dysfunction and loss of PDGFRB pericyte marker in the precuneus and parietal white matter in Alzheimer's disease. J Cereb Blood Flow Metab. 2018;38:103-15.

40. Zhao P, Feng Z, Qi Q, Huang B, Chen A, Li X, et al. Increased NG2 and SOX2 expression is associated with high-grade choroid plexus tumors. Oncol Lett. 2017;14:1802-6. 
41. Milesi S, Boussadia B, Plaud C, Catteau M, Rousset MC, De Bock F, et al. Redistribution of PDGFRbeta cells and NG2DsRed pericytes at the cerebrovasculature after status epilepticus. Neurobiol Dis. 2014;71:151-8.

42. Bell RD, Winkler EA, Sagare AP, Singh I, LaRue B, Deane R, et al. Pericytes control key neurovascular functions and neuronal phenotype in the adult brain and during brain aging. Neuron. 2010;68:409-27.

43. Castejon OJ. Ultrastructural pathology of cortical capillary pericytes in human traumatic brain oedema. Folia Neuropathol. 2011;49:162-73.

44. Zehendner CM, Sebastiani A, Hugonnet A, Bischoff F, Luhmann HJ, Thal SC. Traumatic brain injury results in rapid pericyte loss followed by reactive pericytosis in the cerebral cortex. Sci Rep. 2015;5:13497.

45. Hill J, Rom S, Ramirez SH, Persidsky Y. Emerging roles of pericytes in the regulation of the neurovascular unit in health and disease. J Neurolmmune Pharmacol. 2014;9:591-605.

46. Niu F, Yao H, Zhang W. Sutliff RL, Buch S. Tat 101-mediated enhancement of brain pericyte migration involves platelet-derived growth factor subunit B homodimer: implications for human immunodeficiency virus-associated neurocognitive disorders. J Neurosci. 2014;34:11812-25.

47. Persidsky Y, Hill J, Zhang M, Dykstra H, Winfield M, Reichenbach NL, et al. Dysfunction of brain pericytes in chronic neuroinflammation. J Cereb Blood Flow Metab. 2016:36:794-807.

Ready to submit your research? Choose BMC and benefit from:

- fast, convenient online submission

- thorough peer review by experienced researchers in your field

- rapid publication on acceptance

- support for research data, including large and complex data types

- gold Open Access which fosters wider collaboration and increased citations

- maximum visibility for your research: over $100 \mathrm{M}$ website views per year

At $\mathrm{BMC}$, research is always in progress.

Learn more biomedcentral.com/submissions 